See Article page 1776.

\section{Commentary: I am not an expert, but I work in the company of experts}

\author{
Riyad Karmy-Jones, MD, FACS, FRCSC, and \\ BeeJay Feliciano, MD, FACS
}

Fukuhara and colleagues ${ }^{1}$ describe in detail an approach for managing chronic type B aortic dissection, either de novo or after ascending/arch repair. As we know and they describe, chronic dissection management can be influenced by stiffness of the septum, which can lead to endocollapse-as well as oversizing to push the septum away-that runs the risk of stent graft-induced new entry (SINE). Further, lack of false lumen compression in the setting of distal endoleaks from fenestrations or branch vessels can frustrate the goal of treatment. Anecdotally, we have found that in the subacute and chronic setting a useful assessment is intravascular ultrasound and gentle hand inflation with a balloon to test how much the septum moves to gauge its rigidity. Septotomy with or without wire fenestration to manage these issues has, as the authors note, been described before, including open and catheter-based approaches. By allowing the endograft to expand across the true and false lumen, the benefit based on the authors' experience suggests that in the short- to intermediate-term the risk of persistent endoleaks and SINE is decreased significantly. Some complications the authors describe include enfolding of the septum after wire septotomy with need for more distal intervention and with proximal laser septotomy retrograde SINE, albeit single patients only. It is of note that several patients required management of branch vessels with bypass, stenting, or fenestration. I think some of us would be wary of the

\footnotetext{
From the Division of Thoracic and Vascular Surgery, PeaceHealth Southwest Washington Medical Center, Vancouver, Wash.

Disclosures: The authors reported no conflicts of interest.

The Journal policy requires editors and reviewers to disclose conflicts of interest and to decline handling or reviewing manuscripts for which they may have a conflict of interest. The editors and reviewers of this article have no conflicts of interest.

Received for publication Aug 2, 2021; revisions received Aug 2, 2021; accepted for publication Aug 3, 2021; available ahead of print Aug 8, 2021.

Address for reprints: Riyad Karmy-Jones, MD, FACS, FRCSC, Division of Thoracic and Vascular Surgery, PeaceHealth Southwest Washington Medical Center, 505 NE 87th Ave, Bldg B, Suite 301, Vancouver, WA 98664 (E-mail: Rkarmy-jones@peacehealth.org).

J Thorac Cardiovasc Surg 2023;165:1787

$0022-5223 / \$ 36.00$

Copyright (c) 2021 by The American Association for Thoracic Surgery

https://doi.org/10.1016/j.jtcvs.2021.08.005
}

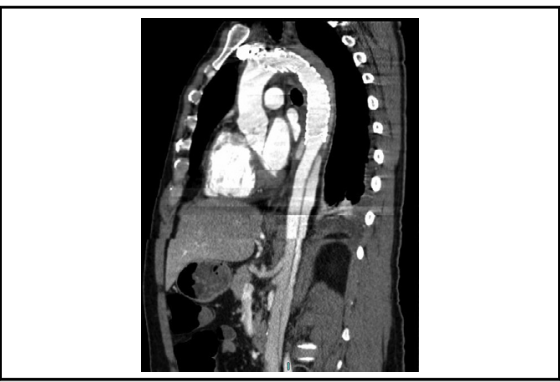

Residual distal endoleaks after TEVAR for acute type $B$ dissection mesenteric ischemia.

CENTRAL MESSAGE

Septotomy in expert hands is a useful tool for managing chronic type $\mathrm{B}$ dissection with reduced risk of SINE and retrograde endoleaks.

long-term influence of proximal septotomy in the setting of hemiarch repair because the natural history of the residual aorta is not clear, but given the close follow-up the team performs, in their hands this seems a reasonable approach. There has been an increasing body of work attempting to define what constitutes an aortic center of excellence and which procedures are optimal for both management of acute dissections to prevent distal complications as well a chronic type B dissection, including residual disease. For instance, is individual surgeon experience more important than hospital volume? It seems reasonable to support ongoing center-specific approaches, with ongoing comparison and cooperation to national and international databases so that the cardiovascular and vascular care community can assess results and make the best choice for the patient based on institutional experience, capabilities, and expertise.

\section{Reference}

1. Fukuhara A, Khaja MS, Williams DM, Marko X, Yang B, Patel HJ, et al. Aortic septotomy to optimize landing zones during thoracic endovascular repair for chronic type B aortic dissection. J Thorac Cardiovasc Surg. 2023;165:1776-86. 